Fixed Point Theory, 22(2021), No. 1, 219-230

DOI: $10.24193 /$ fpt-ro.2021.1.15

http://www.math.ubbcluj.ro/ nodeacj/sfptcj.html

\title{
THE CAUCHY PROBLEM IN SCALE OF BANACH SPACES WITH DEVIATING VARIABLES
}

\author{
NGUYEN BICH HUY* AND PHAM VAN HIEN** \\ *Ho Chi Minh City University of Education, Vietnam \\ E-mail: huynb@hcmue.edu.vn \\ ** Faculty of Applied Sciences, HCMC University of Technology and Education, Vietnam \\ E-mail: hienpv@hcmute.edu.vn
}

\begin{abstract}
In this paper, we first prove the existence and uniqueness results for the Cauchy problems in a scale of Banach spaces with deviating variables of the form $u^{\prime}(t)=F[t, A(t, u(t)), B(u(h(t)))]$. We then apply it to study a Cauchy problem for PDEs in a Gevrey class with deviation at the derivatives. This extends some known results.

Key Words and Phrases: Scale of Banach spaces, Cauchy problem, deviating variable, Gevrey function, fixed point.
\end{abstract}

2020 Mathematics Subject Classification: 35A10, 34G20, 58D25, 47H10.

Acknowledgment. The authors are very grateful to the referees for their careful reading the work that improve the paper. The paper was written when the authors were visiting the Vietnam Institute for Advanced Study in Mathematics (VIASM). The authors thank the institute for its hospitality. This paper is funded by Vietnam National Foundation for Science and Technology Development (NAFOSTED) under grant number 101.02-2019.327.

\section{REFERENCES}

[1] K. Asano, A note on the abstract Cauchy-Kowalewski theorem, Proc. Japan Acad. Ser. A, 64(1998), 102-105.

[2] A. Augustynowicz, H. Leszczyński, W. Walter, Cauchy-Kovalevskaya theory for nonlinear equations with deviating variables, Nonlinear Analysis, 45(2001), 743-753.

[3] M.S. Baouendi, C. Goulaouic, Remark on the abstract form of nonlinear Cauchy-Kovalevsky theorems, Comm. Partial Differential Equations, 2(1977), 1151-1162.

[4] E.A. Barkova, P.P. Zabreiko, Fractional differential equations with worsening right-hand sides, Differential Equations, 46(2010), 208-213.

[5] R.F. Barostichi, A.A. Himonas, G. Petronilho, Autonomuos Ovsyanniikov theorem and applications to nonlocal evolution equations and systems, J. Funct. Anal., 270(2016), 330-358.

[6] H. Begehr, Eine Bemerkung zum nichtlinearen klassichen Satz von Cauchy-Kowalewsky, Math. Nachr., 131(1987), 175-181.

[7] R.E. Caflish, J. Lowengrub, Convergence of the vortex method for vortex sheets, SIAM J. Numer. Anal., 26(1989), 1060-1080. 
[8] K. Deimling, Ordinary Differential Equations in Banach Spaces, Lect. Notes Math., 596(1977), Springer, Berlin.

[9] D. Finkelshtein, Around Ovcyannikov's method, Methods Funct. Anal. Topology, 21(2015), 131-150.

[10] D. Finkelshtein, Y. Kondratiev, Y. Kozitsky, Glauber dynamics in continuum: a constructive approach to evolution of states, Discrete Continuous Dynam. Systems - A, 33(2013), 1431-1450.

[11] M. Ghisi, The Cauchy-Kowalevsky theorem and noncompactness measure, J. Math. Sci. Univ. Tokyo, 4(1994), 627-647.

[12] N.B. Huy, N.A. Sum, N.A. Tuan, A second-order Cauchy problem in a scale of Banach spaces and application to Kirchhoff equations, J. Diff. Eq., 206(2004), 253-264.

[13] M. Kawagishi, T. Yamanaka, On the Cauchy problem for PDEs in the Gevrey class with shrinking, J. Math. Soc. Jpn., 54(2002), 649-677.

[14] M. Kawagishi, T. Yamanaka, The Heat equation and the shrinking, EJDE, 97(2003), 1-14.

[15] M.C. Lambardo, M. Canone, M. Sammartino, Well-posedness of the boundary layer equations, SIAM J. Math. Anal., 35(2003), 987-1004.

[16] Y. Maekava, On the inviscid limit problem of the vorticity equations for viscous incompressible flows in the half plane, Comm. Pure Appl. Math., 67(2014), 1045-1128.

[17] L. Nirenberg, An abstract form of the nonlinear Cauchy-Kowalewski theorem, J. Differential Geom., 6(1972), 561-576.

[18] T. Nishida, A note on a theorem of Nirenberg, J. Diff. Geometry, 12(1977), 629-633.

[19] T. Nishida, Fluid dynamical limit of the nonlinear Boltzmann equation to the level of the compressible Euler equation, Comm. Math. Phys., 61(1978), 119-148.

[20] L.V. Ovcyannikov, Singular operators in a scale of Banach spaces, Soviet Math Dokl., 163(1965), 819-822.

[21] L.V. Ovcyannikov, A nonlinear Cauchy problem in a scale of Banach spaces, Soviet. Math. Dolk., 12(1971), 1497-1502.

[22] M. Reissig, The existence and uniqueness of analytic solutions for amoving boundary problem for Hele-Shaw flows in the plane, Nonlinear Anlysis: Methods \& Applications, 23(1994), 565576.

[23] M.V. Safonov, The abstract Cauchy-Kowalevskaya theorem in a weighted Banach space, Comm. Pure Appl. Math., VXLVIII(1995), 629-637.

[24] M. Sammartino, R.E. Caflish, Zero viscosity limit for analytic solutions of the Navier-Stokes equation on half-space, I. Existence for Euler and Prandtl equations, Comm. Math. Phys., 192(1998), 433-461.

[25] F. Treves, An abstract nonlinear Cauchy-Kowalewska theorem, Trans. Amer. Math. Soc., 150(1970), 72-92.

[26] W. Tutschke, Initial value problem for generalized analytic functions depending on time (an extension of theorems of Cauchy-Kovalevskaya and Holmgren), Soviet. Math. Dolk., 25(1982), 201-205.

[27] T. Yamanaka, Note on Kowalewskaya system of partial differential equations, Comm. Math. Univ. St Paul, 9(1960), 7-10.

[28] T. Yamanaka, M. Kawagishi, A Cauchy-Kowalevskaya type theory in the Gevrey class for PDEs with shrinking, Nonlinear Analysis, 64(2006), 1860-1884.

Received: January 29, 2019; Accepted: January 28, 2020. 